\title{
SITUAÇÃO DOS RECURSOS HÍDRICOS NAS BACIAS HIDROGRÁFICAS DOS RIOS PIRACICABA, CAPIVARI E JUNDIAÍ UTILIZANDO MODELO DESENVOLVIDO EM DINÂMICA DE SISTEMAS
}

\author{
RODRIGO M. SÁNCHEZ-ROMÁN ${ }^{1}$, MARCOS V. FOLEGATTI², \\ ALBA M. G. ORELLANA-GONZÁLEZ ${ }^{3}$
}

\begin{abstract}
RESUMO: Utilizou-se modelo desenvolvido em dinâmica de sistemas, especificamente para as Bacias Hidrográficas dos Rios Piracicaba, Capivari e Jundiaí (BH-PCJ), com cinco simulações para 50 anos de horizonte, como ferramenta para auxiliar na gestão dos recursos hídricos. O modelo estimou as ofertas e demandas de água, e a geração de águas residuárias dos diversos consumidores existentes nas BH-PCJ. Realizou-se simulação utilizando-se das taxas de consumo e de oferta existentes em 2004, e as precipitações com os valores médios constantes. Sob essas premissas, foi encontrado que as demandas de água aumentarão cerca de $76 \%$, que aproximadamente $39 \%$ do volume de água disponível terá origem no reúso das águas residuárias, a carga contaminante aumentará em $91 \%$. O Índice de Falkenmark mudará de $1.403 \mathrm{~m}^{3}$ habitante ${ }^{-1}$ ano-1, em 2004, para $734 \mathrm{~m}^{3} \mathrm{hab}^{-1}$ ano $^{-1}$ em 2054; e o Índice de Sustentabilidade de 0,44 para 0,20. Foram explorados outros quatro cenários: com fator de mudanças nas precipitações anuais de 90 e 110\%; considerando vazão ecológica equivalente a $30 \%$ da vazão média diária, e sem nenhuma mudança nas taxas dos outros fatores, somente na vazão ecológica e no consumo domiciliar de água. Todos eles mostraram tendência à futura crise nos recursos hídricos nas BH-PCJ.
\end{abstract}

PALAVRAS-CHAVE: recursos hídricos, modelagem, simulação.

\section{WATER RESOURCES SITUATION AT PIRACICABA, CAPIVARI AND JUNDIAÍ WATERSHEDS USING A DYNAMIC SYSTEMS MODEL}

\begin{abstract}
Using a dynamic systems model specifically developed for Piracicaba, Capivari and Jundiaí River Water Basins (BH-PCJ) as a tool to help to analyze water resources management alternatives for policy makers and decision takers, five simulations for 50 years timeframe were performed. The model estimates water supply and demand, as well as wastewater generation from the consumers at BH-PCJ. A run was performed using mean precipitation value constant, and keeping the actual water supply and demand rates, the "business as usual" scenario. Under these considerations, it is expected an increment of about $\sim 76 \%$ on water demand, that $\sim 39 \%$ of available water volume will come from wastewater reuse, and that waste load increases to $\sim 91 \%$. Falkenmark Index will change from $1,403 \mathrm{~m}^{3}$ person $^{-1}$ year $^{-1}$ in 2004 , to $734 \mathrm{~m}^{3} \mathrm{P}^{-1}$ year ${ }^{-1}$ by 2054 , and the Sustainability Index from 0.44 to 0.20 . Another four simulations were performed by affecting the annual precipitation by 90 and 110\%; considering an ecological flow equal to $30 \%$ of the mean daily flow; and keeping the same rates for all other factors except for ecological flow and household water consumption. All of them showed a tendency to a water crisis in the near future at BH-PCJ.
\end{abstract}

KEYWORDS: water resources, modeling, simulation.

\footnotetext{
${ }^{1} E^{2}{ }^{0}$ em Irrigação e Drenagem, Pós-Doutorando no Departamento de Engenharia Rural, Escola Superior de Agricultura "Luiz de Queiroz", Universidade de São Paulo, Av. Pádua Dias 11, Piracicaba - SP, Fone: (0XX19) 3447-8569, Fax: (0XX19) 3435-8571, Bolsista CT-HIDRO PDJ/CNPq, rmsroman@esalq.usp.br

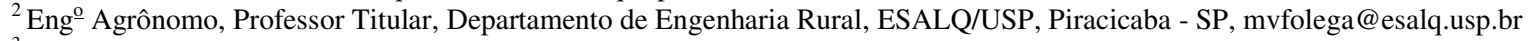

${ }^{3}$ Economista, Bolsista da FAPESP, Doutoranda no Departamento de Engenharia Rural, ESALQ/USP, Piracicaba - SP, gonzalez@esalq.usp.br

Recebido pelo Conselho Editorial em: 12-2-2008

Aprovado pelo Conselho Editorial em: 23-9-2009
}

Eng. Agríc., Jaboticabal, v.29, n.4, p.578-590, out./dez. 2009 


\section{INTRODUÇÃO}

A problemática da água tem diferentes dimensões: a) a população rural tem dificuldade de obtenção de água em volume e em qualidade, enquanto a população urbana recebe água tratada pelas empresas de saneamento; b) o nível de renda das pessoas influencia na oportunidade de uso desse recurso; c) a escassez da água sofre interferência quanto à escala de consumo; d) a disponibilidade do recurso hídrico manifesta-se de forma qualitativa e quantitativa, tanto no espaço quanto no tempo em uma mesma região; e) a água é um recurso essencial para a vida. São muitas as perspectivas, interesses, percepções e alternativas de uso dos recursos hídricos, dada as diversidades de usuários, situações, rendas, prioridades e localizações.

A importância na gestão da água está diretamente ligada com a questão da sustentabilidade ambiental, sendo uma função do desenvolvimento dos diversos componentes políticos, econômicos e sociais atuantes dentro da bacia hidrográfica, e da sensibilidade desses componentes com o tema da gestão integrada dos recursos hídricos. O aumento na renda das populações urbana e rural possibilitará melhoria no padrão de vida e, consequentemente, a demanda de água crescerá. Adicionalmente, o crescimento natural da população implica aumento substancial na demanda de água e de alimentos, e na contaminação dos corpos de água. $\mathrm{O}$ foco da análise das simulações realizadas é o setor agropecuário, por ser o responsável pela produção de alimentos.

Tudo isso leva ao seguinte questionamento: até onde é possível manter o crescimento das atividades produtivas nos diversos setores da economia e ainda satisfazer as demandas crescentes da população sem prejudicar a sustentabilidade dos recursos hídricos. Esse questionamento deverá ser respondido através do Modelo de Gestão de Recursos Hídricos das Bacias Hidrográficas dos Rios Piracicaba, Capivari e Jundiaí (MRH-PCJ). Essas bacias têm sido emblemáticas e precursoras das políticas do Plano Nacional de Gestão de Recursos Hídricos no atual Sistema Nacional de Recursos Hídricos do Brasil, que, com o apoio da Agência Nacional de Águas e em articulação com os Comitês de Bacia Hidrográfica (CBH) do PCJ, instituíram o Plano de Bacias e a cobrança pelo uso dos recursos hídricos de domínio da União.

\section{Recursos hídricos e enfoque sistêmico}

Existe uma relação direta entre a gestão de recursos hídricos e o enfoque sistêmico, uma vez que os fatores que afetam os recursos hídricos são sistêmicos e não lineares (OLHSSON \& TURTON, 1999). A teoria dos sistemas é a base desses estudos. O princípio elementar do estudo sistêmico é o da conectividade. Entende-se sistema como um conjunto de elementos com ligações entre si e o ambiente. Cada sistema compõe-se de subsistemas, que fazem parte de um conjunto maior, sendo cada subsistema independente e ao mesmo tempo aberto e inter-relacionado com outros subsistemas (SANTOS, 1982).

Utiliza-se na gestão dos recursos hídricos o enfoque sistêmico na análise e no seu aperfeiçoamento. As unidades que compõem o sistema de recursos hídricos de uma bacia são analisadas como um sistema dinâmico e complexo dentro dos limites físicos de uma determinada bacia. Na bacia, podem coexistir atividades múltiplas e explorações que interagem com insumos, tais como: adubos, defensivos e geração de produtos agrícolas, energia, resíduos e contaminantes de origem agrícolas, industriais, urbanos, etc. (COSTA, 1993). Mas, a sustentabilidade dos recursos hídricos deve estar vinculada à capacidade dos usuários desses recursos de conservarem ou aumentarem sua qualidade de vida, mantendo e garantindo esses recursos para as próximas gerações.

\section{Dinâmica de sistemas: simulação e análise}

A metodologia da Dinâmica de Sistemas (DS) é fundamentada e derivada da Teoria de Controle de Servomecanismos, que foi desenvolvida por FORRESTER (1961). O princípio fundamental dessa metodologia é que todo comportamento dinâmico é consequência da estrutura do sistema (POWERSIM, 1996). Esse caracteriza-se por apresentar mudanças ao longo do tempo. 
Podem ser incluídos sistemas econômicos, biológicos, sociais, psicológicos, gerenciais, ecológicos e todos aqueles que manifestam processos de retroalimentação.

Segundo RICHARDSON (1991), as principais características da metodologia de DS são: a) definição dos problemas dinamicamente; b) enfoque nas características intrínsecas do sistema; c) conceituação do sistema real por meio da interconexão contínua de círculos de retroalimentação e de causalidade; d) identificação de estoques e fluxos de entrada e saída; e) formulação de um modelo comportamental, capaz de reproduzir a dinâmica do problema; f) entendimento e esclarecimento derivado de mudanças políticas efetuadas no modelo e seus consequentes resultados; e g) implementação de mudanças e novas políticas com base nesses entendimentos.

Os modelos de simulação dinâmica são descrições abstratas do mundo real que permitem representar problemas complexos caracterizados por sua dinâmica não linear, relações de retroalimentação e defasagens no tempo e no espaço (WIAZOWSKI et al., 1999). Um modelo de simulação dinâmica deve capturar somente os fatores essenciais de um sistema real e deve abstrair-se dos demais fatores. O uso principal dos modelos é o de comunicar um ponto de vista do mundo que procura aproximar-se da realidade, tentando compreender um problema específico e visando a predizer o seu comportamento (PÉREZ MAQUEO et al., 2006). O usuário deve estar sempre consciente das limitações do modelo que está utilizando.

Assim, baseados nas experiências em outros países e outras bacias hidrográficas, pretende-se avaliar o estágio de desenvolvimento da BH-PCJ e analisar os prognósticos estimados pelo MRHPCJ.

\section{MATERIAL E MÉTODOS}

O pensamento sistêmico envolve mudanças de paradigmas sobre como as coisas funcionam. Esta metodologia facilita a visualização das inter-relações entre os elementos dos sistemas, identifica soluções para os problemas em longo prazo, procura pontos onde pequenas mudanças ocasionarão efeitos positivos ao sistema e evitam soluções que tratam apenas dos sintomas dos problemas (POWERSIM, 1996). A utilidade dos modelos decorre da impossibilidade de realizar experimentos com o sistema real.

O MRH-PCJ foi desenvolvido na plataforma STELLA 9.0. Com esse software, interconectaram-se os elementos ambientais, físicos, sociais e econômicos que explicam a dinâmica de comportamento, tanto da oferta como da demanda de recursos hídricos, bem como a geração de águas residuárias dos diversos usuários existentes nas BH-PCJ. O modelo é uma ferramenta para auxiliar os gestores (pessoas responsáveis pelas formulações de políticas e tomadas de decisões) quanto às diversas alternativas para a gestão dos recursos hídricos dentro do CBH-PCJ.

Realizaram-se cinco simulações com horizonte de tempo de 50 anos (Tabela 1). Considerou-se o volume das precipitações anuais iguais ao valor médio de $1.460 \mathrm{~mm} \mathrm{ano}^{-1}$ (IRRIGART, 2004), no cenário "Business as Usual" (BaU) ou afetadas por mudanças climáticas em 90 e 110\% desse valor, nos cenários que têm a ver com mudanças climáticas, considerando vazão ecológica equivalente a 1/40 da vazão básica. Finalmente, nos últimos dois cenários, com vazão ecológica igual a $19,2 \mathrm{~m}^{3} \mathrm{~s}^{-1}$ (30\% da vazão básica) e sem nenhuma mudança nas taxas das outras variáveis, somente no volume consumido nas residências urbanas e rurais (Figura 1). 
TABELA 1. Cenários avaliados utilizando o Modelo de Gestão de Recursos Hídricos das Bacias Hidrográficas dos Rios Piracicaba, Capivari e Jundiaí (MRH-PCJ) num horizonte de 50 anos. Evaluated scenarios using the Water Resources Model for the Piracicaba, Capivari, and Jundiaí River Basins for a 50-year time frame.

\begin{tabular}{cll}
\hline Cenário & \multicolumn{1}{c}{ Classe } & \multicolumn{1}{c}{ Descrição } \\
\hline 1 & BaU & Business-as-usual - sem nenhuma mudança \\
2 & Câmbio climático & Diminuição em 10\% das precipitações \\
3 & Câmbio climático & Aumento em 10\% das precipitações \\
4 & BaU+Q Qecol=19,2 $\mathrm{m}^{3} \mathrm{~s}^{-1}$ & BaU + vazão ecológica equivalente a 30\% da vazão básica do rio \\
5 & Combinação de condições & Cenário 4 + mudanças no consumo doméstico \\
\hline
\end{tabular}

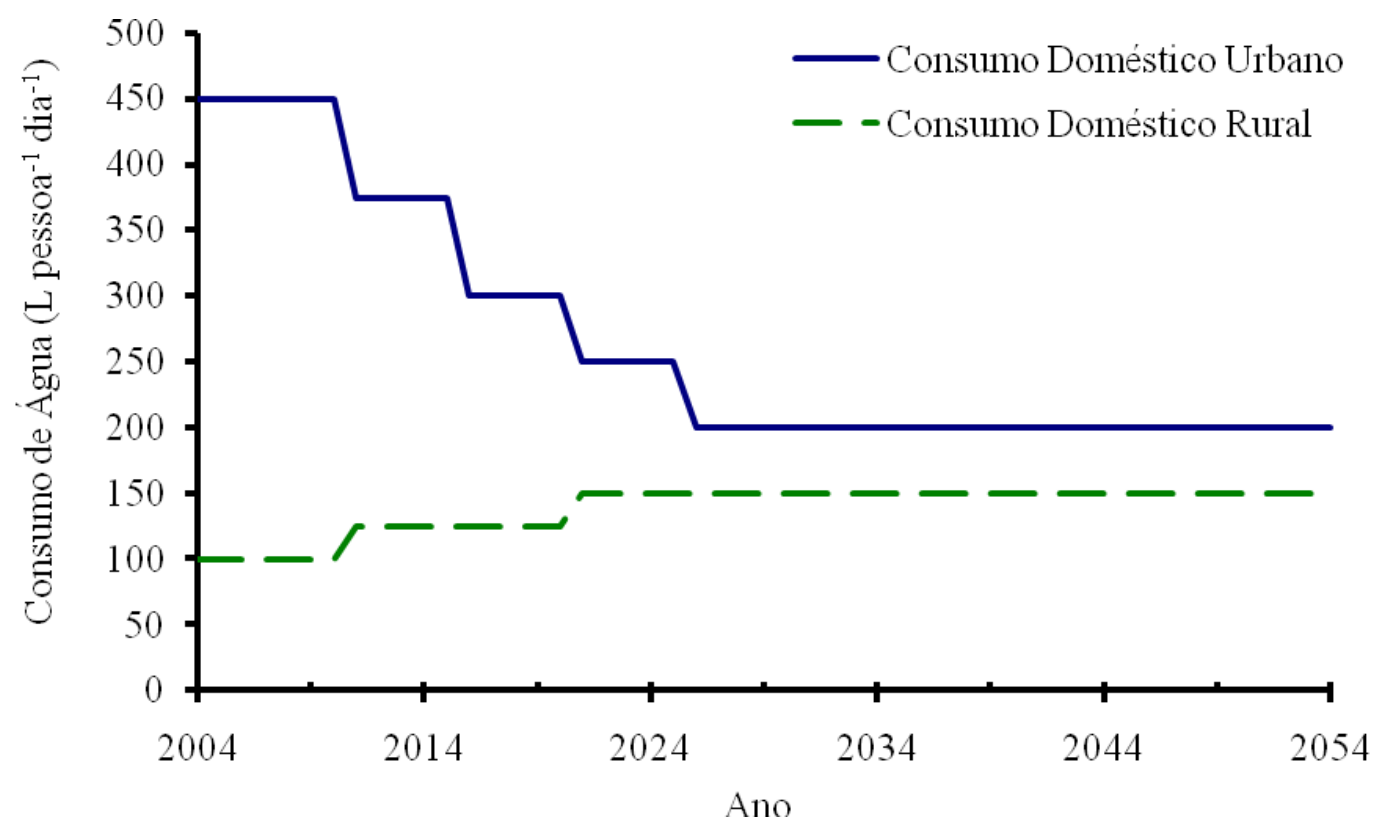

FIGURA 1. Consumo de água nos domicílios urbanos e rurais utilizados no cenário 5. Urban and rural water consumption demand considered for scenario 5.

\section{Demanda de água dos diversos setores}

A definição dos parâmetros das demandas de água tem como base o estudo in situ dos consumos de água pelos principais usuários da região, de estudos realizados e apresentados nos diversos Planos Estaduais, de fontes de informação secundária das agências vinculadas aos setores produtivos, e das instituições que administram o recurso hídrico (PERH, 2004; PERH, 2005; SIRGH, 2007; SABESP, 2007; DAEE, 2007; IEA, 2007; UNICA, 2007).

Para ser efetiva e apurada, a modelagem da agricultura irrigada e da criação de animais precisa ter o conhecimento detalhado das condições de uso dos recursos hídricos, que, por sua vez, depende do cadastro dos usuários para todas as atividades e do uso que os mesmos fazem da água. Assim, devem ser levantados dados nas sub-bacias no tocante ao cadastro de irrigantes, à qualidade das águas superficiais e à vazão correspondente ao $Q_{7}^{10}$. A existência de lacunas na informação cria algumas incertezas nos consumos e nos índices usados durante a simulação.

No caso da vazão ecológica, foram revisadas diversas metodologias para definir o valor a ser usado na simulação. Optou-se por usar, no cenário $\mathrm{BaU}$, a que proporcionava a menor vazão ecológica, já que o processo para encontrar um valor que permita melhorar o habitat do rio compete ao CBH-PCJ. Aplicando o método utilizado na França para esquemas existentes (SOUCHON \& 
KEITH, 2001), deveria ser utilizado 1/40 do valor da vazão básica. Segundo o PERH (2005), a vazão básica é $64 \mathrm{~m}^{3} \mathrm{~s}^{-1}$; portanto, a vazão ecológica utilizada no MRH-PCJ é 1,6 $\mathrm{m}^{3} \mathrm{~s}^{-1}$.

O MRH-PCJ utiliza para analisar o impacto dos diversos setores na sustentabilidade dos recursos hídricos, o Índice de Sustentabilidade (XU et al., 2002) que considera a relação entre a disponibilidade de água e a oferta de água, e a relação entre água disponível total e o número de habitantes na bacia para cada ano (FALKENMARK, 1989; FALKENMARK et al., 2007) para avaliar a situação existente ao longo do tempo na BH-PCJ.

\section{Caracterização da região de estudo}

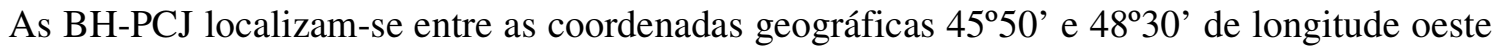
e $22^{\circ} 00^{\prime}$ e $23^{\circ} 20^{\prime}$ de latitude sul. As bacias fazem parte da bacia do Rio Tietê em sua porção média. A bacia do Rio Piracicaba apresenta desnível topográfico de cerca de $1.250 \mathrm{~m}$ em extensão aproximada de 250 km; a do Rio Capivari é de $250 \mathrm{~m}$ em extensão de $180 \mathrm{~km}$, e a do Rio Jundiaí de $500 \mathrm{~m}$ em cerca de $110 \mathrm{~km}$ (PERH, 2005). Por sua posição geográfica, as BH-PCJ encontram-se sob a influência das massas de ar Tropical Atlântica e Tropical Continental, além da massa Polar Atlântica, apresentando diferenças locais que são relacionadas principalmente ao relevo e a distância com relação ao mar (PERH, 2005).

O regime pluviométrico é tipicamente tropical, com período chuvoso entre outubro e abril, e período de estiagem entre maio e setembro, variando, localmente, o início e o término da cada um dos períodos. Nas BH-PCJ, a precipitação média anual varia entre $1.300 \mathrm{~mm}$ e $1.600 \mathrm{~mm}$ (PERH, 2005), e a evaporação potencial anual varia entre 650 mm e 800 mm (PBH, 2007).

Os usos predominantes do solo são as pastagens $(39,06 \%)$ seguidas pelo plantio de cana-de-açúcar $(33,61 \%)$, essa com maior concentração em áreas de menor declividade. O reflorestamento também é atividade significativa em algumas sub-bacias, como a do Rio Jundiaí. As matas nativas concentram-se às margens dos cursos d'água e em unidades de conservação representando apenas 7,93\% da área das BH-PCJ (PERH, 2005).

As três bacias componentes do sistema BH-PCJ têm área de $15.414 \mathrm{~km}^{2}$, abrangendo 64 municípios, sendo 60 no Estado de São Paulo e os quatro restantes no Estado de Minas Gerais (PERH, 2005). Os três rios (Figura 2) são afluentes do Rio Tietê e pertencem à BH - Rio Tietê. A população estimada na Unidade de Gestão dos Recursos Hídricos 5, pelo PERH (2005), para o ano 2004, é de 4.434.937 habitantes, na área urbana, e de 223.998 habitantes, na área rural. As principais atividades econômicas são de caráter industrial, agropecuário, agroindustrial, mineração, recreação, paisagismo, comércio e serviços.

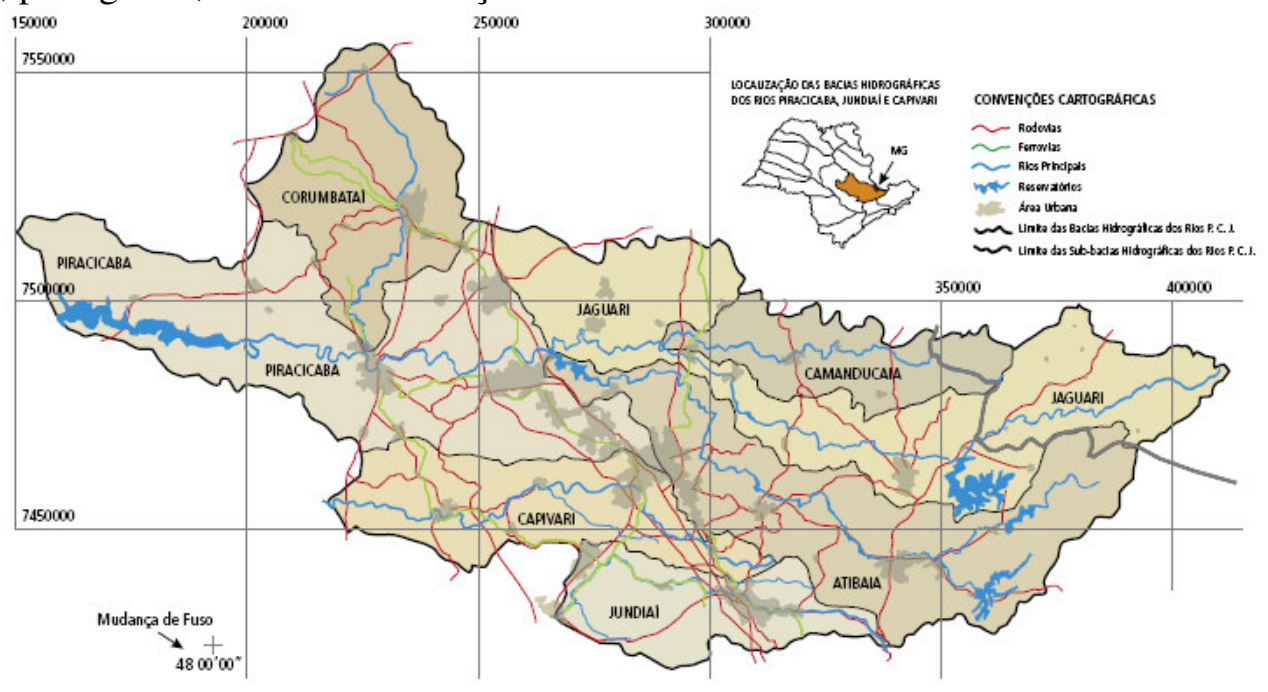

FIGURA 2. Bacias Hidrográficas dos Rios Piracicaba, Capivari e Jundiaí (fonte: PERH, 2005). Piracicaba, Capivari and Jundiaí River Basins. 


\section{Estrutura do sistema de recursos hídricos}

Na Figura 3, representa-se a estrutura do sistema dos recursos hídricos a modelar, para analisar a sustentabilidade do recurso água na região de estudo, em que as variáveis que representam a oferta de água são as águas superficiais e subterrâneas, que garantem o estoque de água no MRH-PCJ. A demanda de água está constituída pelos seguintes requerimentos: população, ambiente, agroindústria, pecuária, indústria e agricultura.

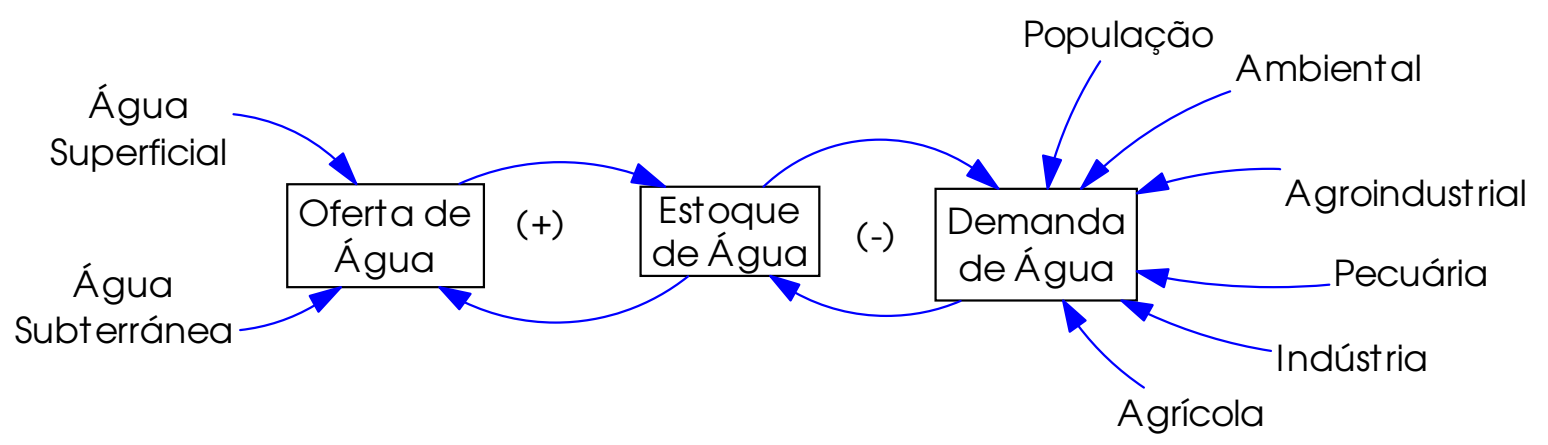

FIGURA 3. Diagrama causal do sistema de recursos hídricos na bacia hidrográfica dos Rios Piracicaba, Capivari e Jundiaí. Causal diagram for the Piracicaba, Capivari and Jundiaí water resources system.

\section{Fontes de informações e dados}

Muitos dos dados utilizados para desenvolver este trabalho são de ordem primária, originados de entrevistas e de coleta de informações de pessoas especializadas no tema e organizações vinculadas à agricultura nos municípios. Outra parte dos dados é de natureza secundária, proveniente de arquivos do Instituto Brasileiro de Geografia e Estatística, do Instituto de Pesquisa Econômica Aplicada, das Prefeituras dos 64 municípios envolvidos, do CBH-PCJ, da Companhia de Saneamento Básico do Estado de São Paulo (estadual e municipal), do Sistema de Informações para o Gerenciamento de Recursos Hídricos do Estado de São Paulo, do Departamento de Águas e Energia Elétrica e da Secretaria de Agricultura e Abastecimento do Estado de São Paulo. Também utilizaram-se estimações médias de requerimentos de água para diferentes culturas agrícolas e espécies animais e informações meteorológicas do Instituto Nacional de Pesquisas Espaciais e do Departamento Nacional de Meteorologia.

\section{Modelo de Gestão dos Recursos Hídricos das Bacias Hidrográficas dos Rios Piracicaba, Capivari e Jundiaí (MRH-PCJ)}

O MRH-PCJ é um modelo de simulação explícito do sistema de Recursos Hídricos das BHPCJ (SÁNCHEZ-ROMÁN \& FOLEGATTI, 2008). A equação do balanço hídrico (1) e a equação da demanda total na BH-PCJ (2), expressas como equação diferencial no MRH-PCJ, são:

$$
\begin{aligned}
& \text { BalanHidric }(t)=\text { BalanHidric }(t-d t)+(\text { OFERTA }- \text { Saída_água_BHPCJ - Demanda_Total_PCJ) } d t \\
& \text { Demanda_Total_PCJ }=\text { VTRAG + VTRAI + VTRamb + VTRInd + VTRpop + VTRPEC + } \\
& \text { (Demanda_RMSP } 86400 \text { 365) }
\end{aligned}
$$

em que,

BalancHidric $(t)$ - balanço hídrico no ano $t, \mathrm{~m}^{3}$ ano $^{-1}$;

OFERTA - oferta de água no ano $t, \mathrm{~m}^{3}$ ano $^{-1}$;

Saída_água_BHPCJ - volume de água descarregado à jusante da Bacia Hidrográfica dos Rios Piracicaba, Capivari e Jundiaí, $\mathrm{m}^{3}$ ano $^{-1}$; 
Demanda_Total_PCJ - volume de água total demandado pelas diversas atividades econômicas na Bacia Hidrográfica dos Rios Piracicaba, Capivari e Jundiaí, $\mathrm{m}^{3}$ ano $^{-1}$;

VTRAG - volume total de água requerido pela agricultura, $\mathrm{m}^{3} \mathrm{ano}^{-1}$;

VTRAI - volume total de água requerido pela agroindústria, $\mathrm{m}^{3} \mathrm{ano}^{-1}$;

VTRamb - volume total de água para garantir a vazão ecológica, $\mathrm{m}^{3} \mathrm{ano}^{-1}$;

VTRInd - volume total de água requerido pela indústria, $\mathrm{m}^{3} \mathrm{ano}^{-1}$;

VTRpop - volume total de água requerido pelas populações rural e urbana, $\mathrm{m}^{3}$ ano $^{-1}$;

VTRPEC - volume total de água requerido pela pecuária, $\mathrm{m}^{3}$ ano ${ }^{-1}$, e

Demanda_RMSP - volume total de água requerido pela Região Metropolitana de São Paulo, $\mathrm{m}^{3}$ ano $^{-1}$.

Dentro do MRH-PCJ, existem 11 setores para modelar o comportamento dos diversos componentes dentro da BH-PCJ. Esses setores estão divididos em três grupos principais: demanda, oferta, cálculos específicos. Dentro do setor das demandas, temos: agricultura, pecuária, agroindústria, ambiental, população e indústria. No setor da oferta, tem-se a oferta de água, e, no setor de cálculos, têm-se: volume de águas residuais retornadas, volume total requerido na BH-PCJ, receitas geradas pela outorga e equivalente populacional.

$\mathrm{Na}$ Figura 4, representa-se a estrutura do sistema de recursos hídricos vinculados à demanda de água do setor agrícola na região de estudo e proposta no MRH-PCJ.

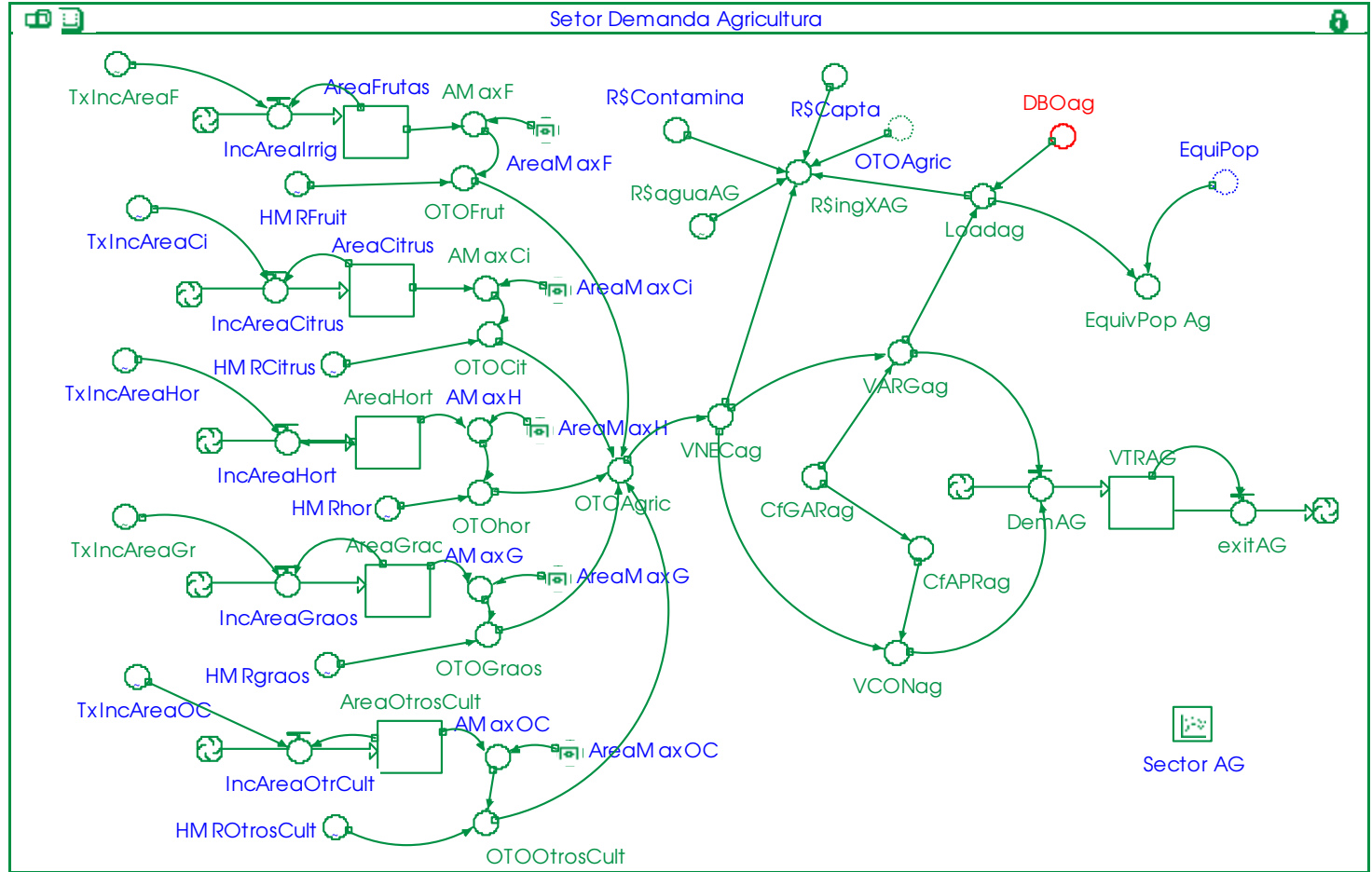

FIGURA 4. Setor demanda de água da Agricultura irrigada no Modelo de Gestão dos Recursos Hídricos da Bacia Hidrográfica dos Rios Piracicaba, Capivari e Jundiaí (MRH-PCJ). Agricultural Sector represented by the Water Resources Model for the Piracicaba, Capivari and Jundiaí River Basins.

A variável que representa a dotação de irrigação é HMRxi $\left(\mathrm{L} \mathrm{s}^{-1} \mathrm{ha}^{-1}\right)$, as áreas por cultivo são Areaxi (ha), e o volume total requerido pela agricultura é VTRAGi $\left(\mathrm{m}^{3} \mathrm{ano}^{-1}\right)$; em que, $i$ representa cada ano analisado, e $x$ o valor particular da variável (cultivo) para cada ano. O volume de água 
necessário leva em consideração a demanda líquida do cultivo mais as ineficiências dos sistemas de irrigação. Está sendo considerado, em cinco grupos, o total de 60 cultivos produzidos na BH-PCJ.

Na Figura 5, representa-se a estrutura do sistema de recursos hídricos vinculados à oferta de água na região de estudo e proposta no MRH-PCJ.

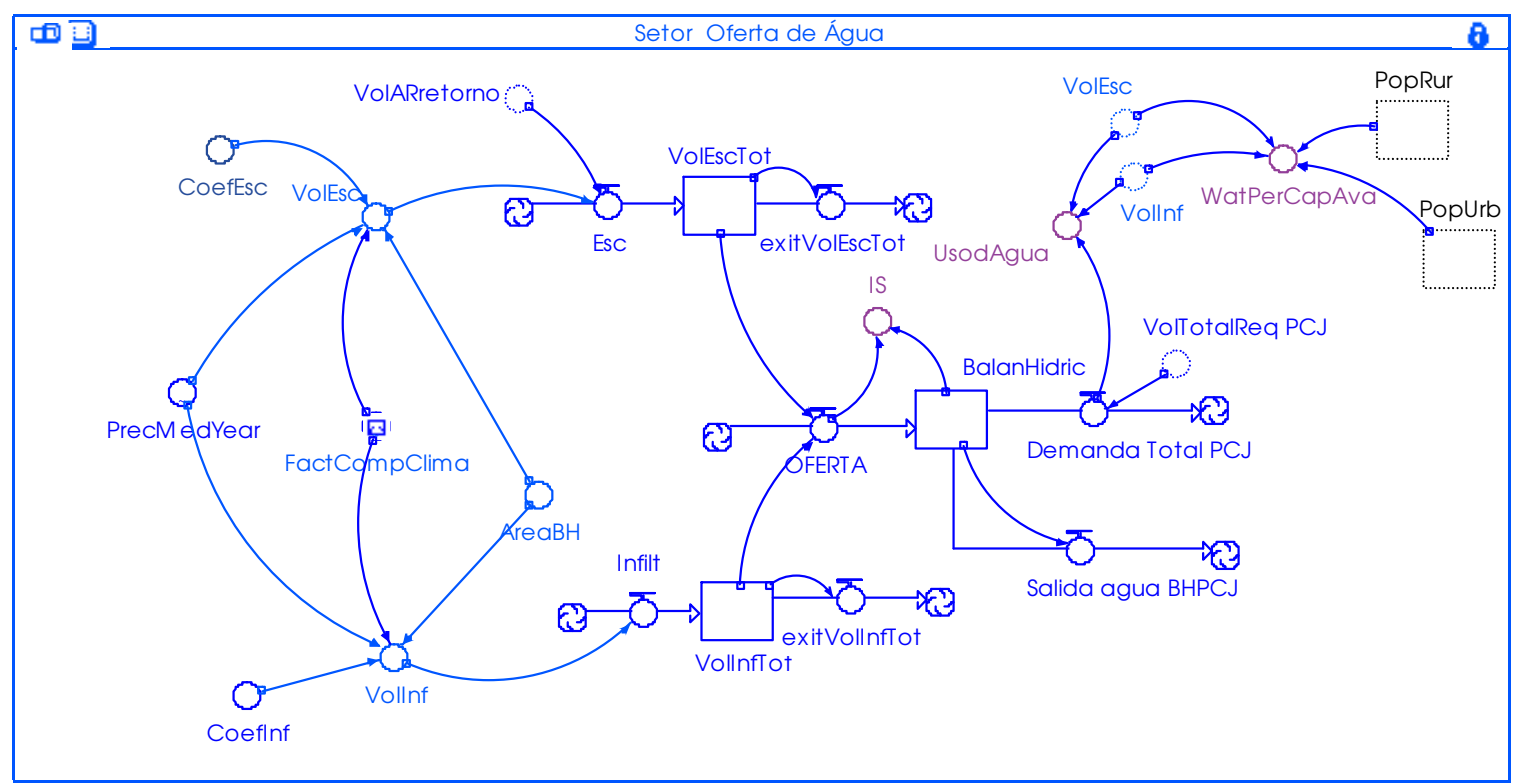

FIGURA 5. Setor oferta de água no Modelo de Gestão dos Recursos Hídricos da Bacia Hidrográfica dos Rios Piracicaba, Capivari e Jundiaí (MRH-PCJ). Water Supply Sector represented by the Water Resources Model for the Piracicaba, Capivari and Jundiaí River Basins.

A variável que representa a oferta de água na BH-PCJ é OFERTA $\left(10^{6} \mathrm{~m}^{3}\right.$ ano $^{-1}=$ $\mathrm{Mm}^{3}$ ano $^{-1}$ ). Os volumes aportados pela chuva são o volume escoado (VolEsc) e o infiltrado (VolInf). Adicionalmente, as águas de retorno (VolARetorno) formam parte da OFERTA, todos em $\mathrm{Mm}^{3}$ ano $^{-1}$. O balanço hídrico (BalanHidric) é realizado, restando a Demanda Total PCJ e as águas que saem à jusante da BH-PCJ (Saída_água_BHPCJ). Com essas informações, são determinados o índice de sustentabilidade (IS), o volume de água disponível per capita anual (WatPerCapAva) e a relação entre o consumo anual pelos diversos demandantes e o volume de água disponível anual (UsodÁgua).

O MRH-PCJ considera que a precipitação acontece uniformemente em toda a bacia hidrográfica, com condições de umidade residual no solo similares e com percentagem de cobertura constante ao longo do horizonte do tempo da simulação. A análise é realizada para a bacia hidrográfica como uma unidade e não discrimina as sub-bacias componentes do sistema BH-PCJ.

O balanço hídrico é realizado considerando valores anuais tanto de oferta quanto de demanda. Os valores de demanda do recurso hídrico e da geração de efluentes são analisados de forma diária, e após esses volumes são anualizados. A oferta é realizada considerando valores anuais de precipitação na região, determinando-se o volume de água disponível.

\section{RESULTADOS E DISCUSSÃO}

Na Tabela 2, pode-se observar que, num período de simulação de 50 anos, mantendo as tendências de consumo e de oferta de água na BH-PCJ no chamado cenário "Business as Usual" (BaU), com time step de um ano, deve-se esperar incremento de aproximadamente $76 \%$ na demanda total de água na área de estudo, ao longo do tempo de simulação. 
Em 2004, a demanda total representava aproximadamente $74 \%$ de todo o volume disponível de água sem considerar as águas de reúso; já em 2007, a relação mudou para aproximadamente 82\%, e espera-se que em 2024 a BH-PCJ seja uma bacia fechada por dois anos, ou seja, todo o volume disponível deverá ser consumido dentro da bacia. Existem previsões de mudanças de consumo (PERH, 2004) que permitirão uma folga nas condições de demanda de água na bacia por uns 6 anos, mas, a partir de 2032, a bacia volta a se fechar, porém, dessa vez, de forma permanente.

A partir desse ponto, o fornecimento de água a novos consumidores somente poderá ser garantido com as águas de reúso, que, para 2054, representarão aproximadamente $39 \%$ das águas disponíveis. O reúso das águas residuárias vai incrementar a oferta de água, mas o volume de água necessário para garantir a diluição das águas residuárias e a sustentabilidade dos ecossistemas dos corpos de água será comprometido pelo aumento do uso das águas residuárias como fonte de abastecimento. Sem dúvida, isso incrementará o preço do tratamento de água, e é preciso prever alternativas para solucionar esse difícil problema.

TABELA 2. Volume escoado, volume infiltrado, volume retornado como água residuária, demanda total e balanço na Bacia Hidrográfica dos Rios Piracicaba, Capivari e Jundiaí simulados para 50 anos, usando o Modelo de Gestão dos Recursos Hídricos da Bacia Hidrográfica dos Rios Piracicaba, Capivari e Jundiaí (MRH-PCJ), no cenário "Business as Usual". Surface runoff, infiltrated volume, effluents returned to river, total demand and balance simulated using the Water Resources Model for the Piracicaba, Capivari and Jundiaí River Basins in a 50-year time frame under the 'Business as Usual' scenario.

\begin{tabular}{cccccc}
\hline Ano & $\begin{array}{c}\text { Volume } \\
\text { Escoado }\end{array}$ & $\begin{array}{c}\text { Volume } \\
\text { Infiltrado }\end{array}$ & $\begin{array}{c}\text { Volume Retornado } \\
\text { como Água Residuária } \\
\text { com }^{3} \text { ano }^{-1}\end{array}$ & Demanda Total & Balanço \\
\hline 2004 & 4.726 & 1.812 & 2.197 & 4.865 & 2.914 \\
2007 & 4.726 & 1.812 & 2.363 & 5.350 & 2.914 \\
2010 & 4.726 & 1.812 & 2.644 & 5.488 & 2.914 \\
2020 & 4.726 & 1.812 & 3.116 & 6.279 & 2.914 \\
2030 & 4.726 & 1.812 & 3.026 & 6.394 & 2.914 \\
2040 & 4.726 & 1.812 & 3.479 & 7.216 & 2.914 \\
2050 & 4.726 & 1.812 & 4.002 & 8.164 & 2.914 \\
2054 & 4.726 & 1.812 & 4.232 & 8.583 & 2.914 \\
\hline
\end{tabular}

Novas estações de tratamento de esgoto e de águas residuárias deverão ser construídas, o que diminuirá a carga contaminante que será despejada nos corpos de água, pois a estimativa é que essa aumentará em $93 \%$ ao longo do período de simulação de 50 anos, se as tendências atuais se mantiverem.

No cenário $B a U$, observou-se que as demandas de água aumentarão em 2030 aproximadamente $24 \%$ em relação às demandas de 2004 , e que cerca de $31 \%$ do volume da água disponível terá origem no reúso das águas residuárias, com o uso total dos recursos hídricos disponíveis de aproximadamente 98\%; a carga contaminante das diversas atividades econômicas terá aumentado cerca de 39\%. Para 2054, as condições serão ainda mais difíceis já que as demandas de água estimadas aumentarão aproximadamente $76 \%$, sendo que em torno de $39 \%$ do volume de água disponível poderá ter origem no reúso das águas residuárias; o uso total dos recursos hídricos disponíveis chegará a aproximadamente $131 \%$ do volume disponível, e a carga contaminante das diversas atividades econômicas terá aumentado a valores superiores a 91\%, comparado com 2004.

Na Figura 6, para o cenário $B a U$, o valor do Índice de Falkenmark que, em 2004, era de $1.403 \mathrm{~m}^{3}$ habitante ${ }^{-1}$ ano $^{-1}$ (713 hab $\mathrm{Mm}^{-3}$ ano $^{-1}$ ) na BH-PCJ, chegará a $1.008 \mathrm{~m}^{3} \mathrm{hab}^{-1}$ ano $^{-1}$ (992 hab Mm${ }^{-3}$ ano $^{-1}$ ) em 2030, e $734 \mathrm{~m}^{3}$ hab $^{-1}$ ano $^{-1}$ (1.363 hab Mm ano $^{-1}$ ) em 2054. Considera-se 
que existe estresse hídrico quando a bacia hidrográfica apresenta índices de 1.000 a $1.600 \mathrm{~m}^{3} \mathrm{hab}^{-1} \mathrm{ano}^{-1}$, e de escassez crônica de água quando o intervalo do volume de água disponível está entre 500 e $1.000 \mathrm{~m}^{3} \mathrm{hab}^{-1}$ ano $^{-1}$ (FALKENMARK, 1989).

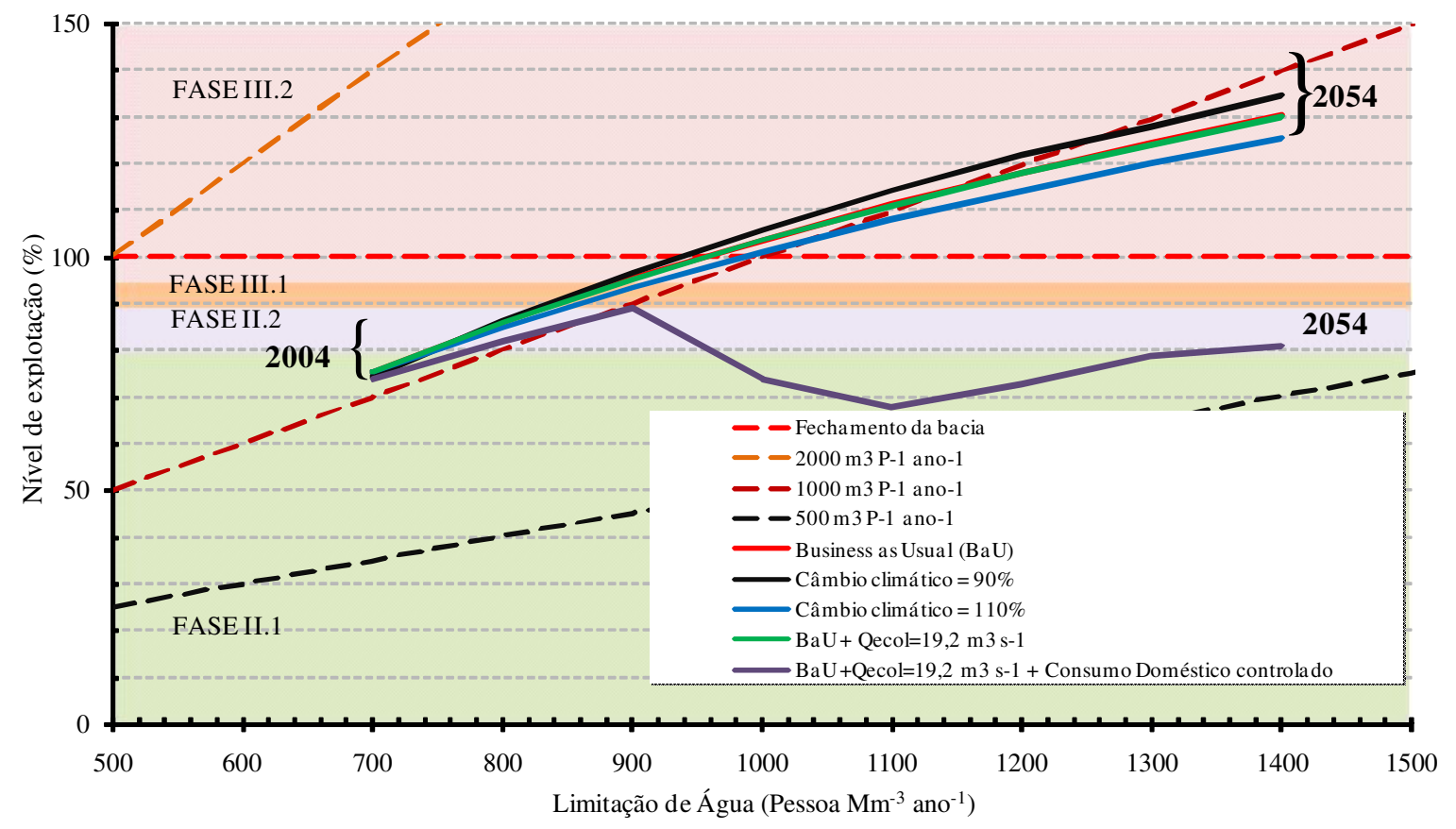

FIGURA 6. Relação entre demanda total de água e disponibilidade de água, em percentagem (no eixo vertical), e densidade populacional por unidade de fluxo, em pessoas por cada $\mathrm{Mm}^{3}$ ano $^{1}$ (na escala horizontal) para a Bacia Hidrográfica dos Rios Piracicaba, Capivari e Jundiaí calculados com o Modelo de Gestão dos Recursos Hídricos da Bacia Hidrográfica dos Rios Piracicaba, Capivari e Jundiaí (MRH-PCJ) para os cinco cenários simulados (Adaptado pelos autores de: FALKENMARK et al., 2007; e KELLER et al., 1998). Relationship between Total Water Demand and Water Availability, in percentage (vertical axis), and Population Density per Unit of Flow, $\mathrm{Mm}^{3}$ ano $^{1}$, (horizontal scale) estimated using the Water Resources Model for the Piracicaba, Capivari and Jundiaí River Basins in a 50-year time frame simulation under five scenarios (Adapted from: FALKENMARK et al., 2007; and KELLER et al., 1998).

Na mesma Figura 6, quando se analisa o desenvolvimento da bacia, segundo KELLER et al. (1998), percebemos que a BH-PCH se encontra em 2007 na Fase II, Fase de Conservação - estágio 1; em outras palavras, precisa diminuir a demanda e incrementar a eficiência no uso da água, mas para 2008, entrará no estágio final da Fase II. Para 2016, entrará na Fase III (Fase de Acrescentamento), que é a fase final de desenvolvimento das bacias hidrográficas, ou seja, fase em que parte da água utilizada deverá ser obtida de trasvases ou da dessalinização dos recursos hídricos de reúso existentes na bacia.

Na Figura 7, apresenta-se o índice de sustentabilidade (IS) proposto por XU et al. (2002) e calculado usando o MRH-PCJ. Para 2004, o IS era de 0,44; chegará a 0,33 em 2030, e será igual a $0,20 \mathrm{em} \mathrm{2054}$. No processo de cálculo do índice, foram consideradas as águas de retorno como parte da oferta das águas disponíveis na BH-PCJ, para assim poder contrastar com os valores encontrados na variável Uso da Água. Quando o valor de IS é maior do que 0,2 indica que existe baixo ou nenhum estresse na oferta de água, mas quando os valores de IS são menores que 0,2 , isso reflete condições de vulnerabilidade dos recursos hídricos. 


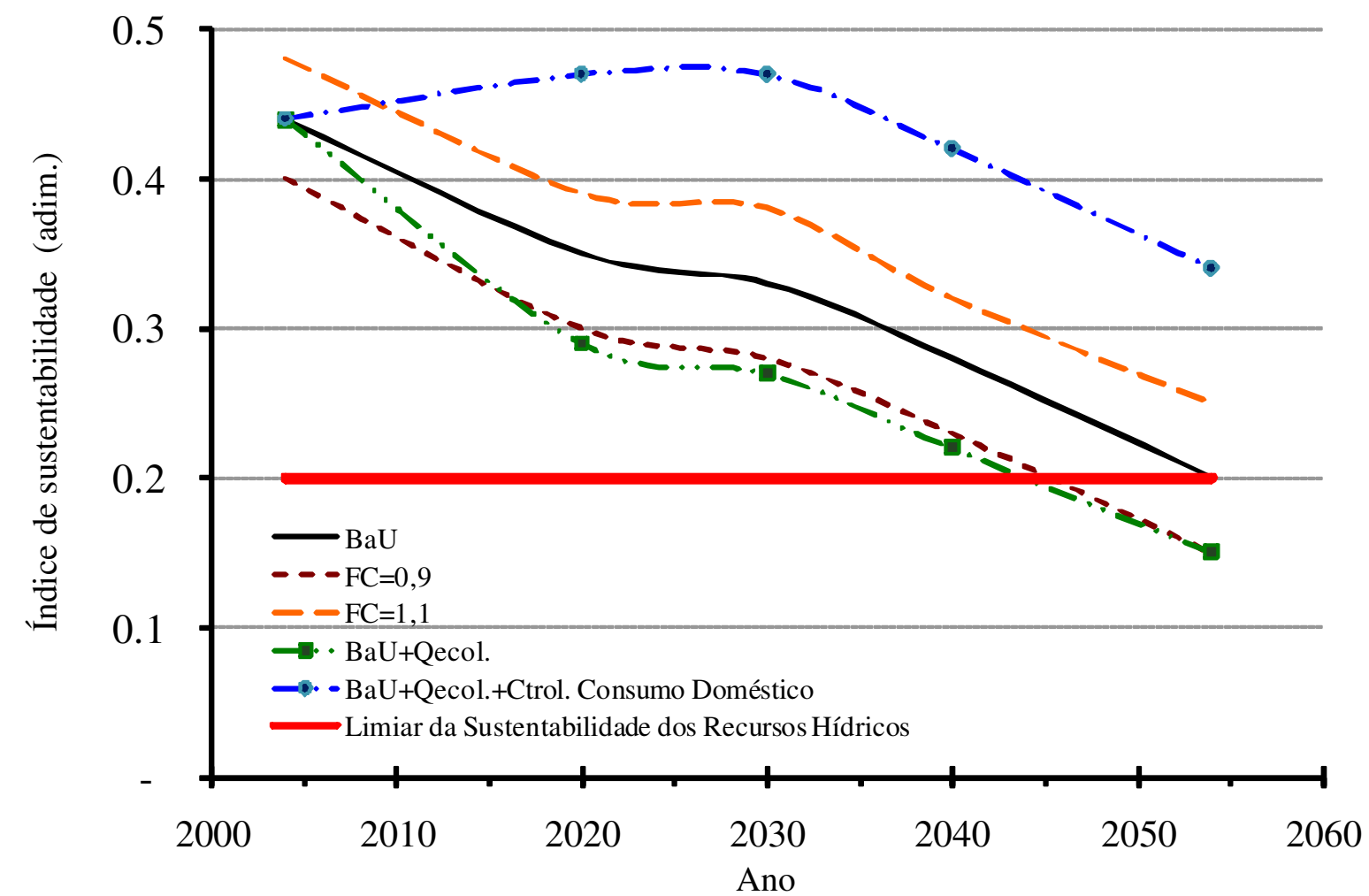

FIGURA 7. Índice de Sustentabilidade (IS - adimensional) proposto por XU et al. (2002), e calculado com o Modelo de Gestão dos Recursos Hídricos da Bacia Hidrográfica dos Rios Piracicaba, Capivari e Jundiaí (MRH-PCJ) para a Bacia Hidrográfica dos Rios Piracicaba, Capivari e Jundiaí. Sustainability Index, dimensionless, proposed by XU et al. (2002), estimated using the Water Resources Model for the Piracicaba, Capivari and Jundiaí River Basins (MRH-PCJ).

Quando analisados os outros cenários simulados, foi encontrado que a tendência à crise é evidente (Figuras 6 e 7) nos três cenários em que não foram realizadas mudanças no manejo dos recursos hídricos; isso significa que nenhum dos usuários foi lesado nas suas demandas de água, ou naqueles cenários onde medidas de proteção do ecossistema e/ou de redução do consumo de água foram consideradas. Considerando que a população é o principal consumidor na BH-PCJ (SÁNCHEZ-ROMÁN et al., 2009), quando foi regulado o uso do recurso hídrico para esses consumidores, as condições de sustentabilidade melhoraram. Isso faz salientar a necessidade imperiosa de reforçar e discutir mais a fundo os processos necessários para garantir uma efetiva e profunda gestão dos recursos hídricos na BH-PCJ.

\section{CONCLUSÕES}

A informação apurada dos diversos consumidores, áreas plantadas, volumes de água residuárias que retorna aos corpos de água ainda é deficiente na bacia e precisa ser elaborada e complementada no menor tempo possível. Isso permitirá melhorar os resultados obtidos pelo modelo de simulação, ajustando cada vez mais o processo da modelagem com a realidade, garantindo que a ferramenta seja um apoio mais útil na tomada de decisões e na busca pela sustentabilidade do sistema de recursos hídricos existente na BH-PCJ.

Todos os resultados encontrados, pelos diversos métodos e índices utilizados, mostram que deverão ser providenciadas medidas adequadas e urgentes que impeçam, ainda mais, a deterioração dos recursos hídricos disponíveis para garantir a sustentabilidade do sistema BH-PCJ. Nos 
próximos 15 anos, a BH-PCJ será uma bacia hidrográfica fechada; para 2030, a situação na bacia será extremamente complicada tal e como indicam os coeficientes calculados; para 2050, a situação será muito próxima de não ser sustentável. Tais cenários mostram a importância de tomadas de decisão em tempo hábil para evitar o caos ecológico e hidrológico na BH-PCJ. Novamente, a crise hídrica é iminente, mas não existe uma percepção geral dessa situação.

\section{AGRADECIMENTOS}

Ao Ministério da Ciência e Tecnologia (MCT), ao Conselho Nacional de Desenvolvimento Científico e Tecnológico (CNPq - Processo 151864/2007-1) e à Fundação de Amparo à Pesquisa do Estado de São Paulo (FAPESP), por meio do Instituto Nacional de Ciência e Tecnologia em Engenharia da Irrigação (INCTEI) e do Processo 2006/60954-4, pelo apoio financeiro a esta pesquisa.

\section{REFERÊNCIAS}

COSTA, M.P.B. Agroecologia: uma alternativa viável às áreas reformadas e à produção familiar. Revista Reforma Agrária, Campinas, v.23, n.1, p.53-69, 1993.

DAEE. DEPARTAMENTO DE ÁGUAS E ENERGIA DO ESTADO DE SÃO PAULO. Disponível em: <http://www.daee.sp.gov.br>. Acesso em: 6 maio 2007.

FALKENMARK, M. The massive water scarcity now threatening Africa - Why isn't it being addressed? Ambio, Estocolmo, v.18, n.2, p.112-118, 1989.

FALKENMARK, M.; BERNTELL, A.; JÄGERSKOG, A.; LUNDQVIST, J.; MATZ, M.; TROPP, $\mathrm{H}$. On the verge of a new scarcity: a call for good governance and human ingenuity. SIWI Policy Brief, Stockholm International Water Institute, 2007. 20 p.

FORRESTER, J.W. Industrial dynamics. Cambridge: M.I.T. Press, 1961. 464 p.

IEA. INSTITUTO DE ECONOMIA AGRÍCOLA. Disponível em: <http://www.iea.sp.gov.br>. Acesso em: 14 maio 2007.

IRRIGART. Relatório da Situação dos Recursos Hídricos das Bacias Hidrográficas dos Rios Piracicaba, Capivari e Jundiaí - 2002/2003. Comitê das Bacias Hidrográficas dos Rios Piracicaba, Capivari e Jundiaí. 2004. v.2, p.126-334. Relatório Final.

KELLER, J.; KELLER, A.; DAVIDS, G. River basin development phases and implications of closure. Journal of Applied Irrigation Science, New York, v.33, n.2, p.145-163, 1998.

OHLSSON, L.; TURTON, A.R. The Turning of a Screw. Social resource scarcity as a bottle-neck in adaptation to water scarcity. Londres: University of London, School of Oriental and African Studies, 1999. 8 p. (SOAS Occasional Paper N.19).

PÉREZ MAQUEO, O.; DELFÍN, C.; FREGOSO, A.; COTLER, H. Modelos de simulación para la elaboración y evaluación de los programas de servicios ambientales hídricos. Gaceta Ecológica, Ciudad de Mexico, n.78, p. 65-84, 2006.

PERH. PLANO ESTADUAL DOS RECURSOS HIDRICOS 2004-2007 DO ESTADO DE SÃO PAULO. Relatório síntese do plano. Piracicaba: Consórcio JMR Engecorps, 2005. 189 p.

PERH. PLANO ESTADUAL DOS RECURSOS HIDRICOS 2004-2007 DO ESTADO DE SÃO PAULO. Sumario executivo. Piracicaba: Consórcio JMR Engecorps, 2004. 25 p.

PLANO DE BACIAS HIDROGRÁFICAS DOS RIOS PIRACICABA, CAPIVARI E JUNDIAÍ PARA O QUADRIÊNIO 2008-2011 (PBH). Piracicaba: STS-Engenharia Ltda., 2007. 655 p.

POWERSIM Co. Introduction to system dynamics. Reston: Powersim Press, 1996. 44 p. 
RICHARDSON, G.P. System dynamics: simulation for policy analysis from a feedback perspective. In: . Modeling for management I: qualitative simulation modeling and analysis. New York: Springer Verlag, 1991. p.144-169.

SABESP. Secretaria de Agricultura e Abastecimento do Estado de São Paulo. Disponível em: <http://www.agricultura.sp.gov.br>. Acesso em: 3 maio 2007.

SÁNCHEZ-ROMÁN, R.M.; FOLEGATTI, M.V. Sustentabilidade da agricultura irrigada nas bacias hidrográficas dos rios Piracicaba, Capivari e Jundiaí: Uma perspectiva em dinâmica de sistemas. Modelo em dinâmica de sistemas para a gestão dos recursos hídricos nas bacias hidrográficas dos rios Piracicaba, Capivari e Jundiaí. 2008. 175 f. Tese (Pós-Doutorado) - Escola Superior de Agricultura "Luiz de Queiroz", Universidade de São Paulo, Piracicaba, 2008.

SÁNCHEZ-ROMÁN, R.M.; FOLEGATTI, M.V.; ORELLANA-GONZÁLEZ, A.M.G. Water Resources Assessment at Piracicaba, Capivari and Jundiaí River Basins: A Dynamic Systems Approach. Water Resources Management, The Netherlands, 2009. Disponível em: $<$ http://dsc.doi.org>. DOI 10.1007/s11269-009-9470-x.

SANTOS, M. O espaço e os seus elementos: questões de método. Revista Geografia e Ensino, Belo Horizonte, v.1, n.1, p.19-30, 1982.

SIRGH. SISTEMA DE INFORMAÇÕES PARA O GERENCIAMENTO DE RECURSOS HÍDRICOS DO ESTADO DE SÃO PAULO. Disponível em: <http://www.sigrh.sp.gov.br/cgibin/bdhm.exe/plu>. Acesso em: 25 abr. 2007.

SOUCHON, Y.; KEITH, P. Freshwater fish habitat: science, management and conservation in France. Aquatic Ecosystem Health and Management Society, Philadelphia, v.4, n.4, p.401-412, 2001.

UNICA. UNIÃO DA INDÚSTRIA DE CANA-DE-AÇÚCAR. Disponível em: $<$ http://www.portalunica.com.br>. Acesso em: 12 maio 2007.

WIAZOWSKI, B.A.; SILVA, C.A.B.; LOURENZANI, W.L. O uso de sistemas dinâmicos como ferramenta de aprendizagem. Economia Rural, Viçosa - MG, v.3, n.10, p. 29-33, 1999.

XU, Z.X.; TAKEUCHI, K.; ISHIDARA, H.; ZHANG, X.W. Sustainability analysis for yellow river water resources using the system dynamics approach. Water Resources Management, The Netherlands, v.16, n.3, p.239-261, 2002. 